\title{
Controlled Fabrication of Highly Monodispersed, Gold Nanoparticles Grafted Polyaniline (Au@PANI) Nanospheres and their Efficient Ammonia Gas Sensing Properties
}

\author{
Abdulla S, Dhakshanamoorthi J, Dinesh VP and Pullithadathil B* \\ Nanosensor Laboratory, PSG Institute of Advanced Studies, Coimbatore, India
}

\begin{abstract}
In the present study, highly monodispersed polyaniline (PANI) and gold nanoparticles grafted PANI nanospheres (Au@PANI) have been fabricated through a facile wet-chemical synthesis process by in-situ polymerization of aniline. The structural characterization of the PANI and Au@PANI nanospheres were carried out by UV-Vis spectroscopy, FT-IR spectroscopy, and High Resolution Transmission Electron Microscopy (HR-TEM). Stable uniform sized nanospheres of PANI and Au@PANI obtained were found to exhibit superior gas sensor performance towards trace-level concentration of ammonia $\left(\mathrm{NH}_{3}\right)$ gas in the range of 1-5 ppm. A comparative analysis of the sensor properties of PANI and Au@PANI nanospheres were performed under ambient conditions. Au@PANI nanospheres exhibited enhanced gas sensing performance with high sensitivity (58.2\%), rapid response (48 s) and recovery (58 s) characteristics (1 ppm of $\mathrm{NH}_{3}$ ). The results indicate the potential use of Au@PANI nanospheres for fabrication of efficient $\mathrm{NH}_{3}$ sensors for varied applications.
\end{abstract}

Keywords: PANI nanospheres; Nanocomposites; Chemiresistors; Gas sensors; $\mathrm{NH}_{3}$ gas monitoring

\section{Introduction}

Polyaniline (PANI), one of the widely used conducting polymers gained its potential place in sensors, photocatalyst, electrochemical industries owing to its unique electrical and redox properties [1]. The existence of polymorphic forms of polyaniline viz., reduced leucoemeraldine form and the fully oxidized pernigraniline form makes them as an interesting class of redox materials for various sensors based application exclusively [2]. Several synthetic strategies have been widely used by researchers and various morphological structures have been developed using template-free, template-based and electrochemical methods [3]. In addition, nanocomposites based on PANI having metal-polymer or metal oxide-polymer hybrid structures also have been shown superior properties and used for fabrication of sensor devices [4,5]. The combination of conducting $\pi$-conjugated polymer network with metal/metal oxide interfaces favour the surface adsorption process of target gas molecules. The localized electronic states in conducting polymers generally make them to behave as $p$-type semiconductors. Due to their redox behaviour of these polymers, the conductivity could be easily altered by doping and de-doping the material [6].

Ammonia is one of the harmful environmental pollutants as-well recognized as a biomarker related to in kidney and liver dysfunctions in the exhaled human breath [7]. Hence, developing room temperature ammonia sensors with enhanced sensitivity and selectivity for device fabrication is highly significant in the current sensor development. So far, there are many reports on PANI based gas sensors exist in literature [8]. But, the sensors based on these materials usually suffer from degradation due to environmental effects and are highly unstable. Issues on the reliability of the polymer based chemosensors are mainly attributed to their polydispersity as well as irregular morphology. In metal nanoparticles integrated PANI nanocomposites, the electron rich polymer matrix acts as a scaffold for the secondary component with high electron density [9]. Introducing metal nanoparticles enhances the selectivity of the sensors and such hybrid materials can be achieved by altering the redox behaviour of polyaniline. These hybrid nanocomposites possess several advantages as high surface area, more active sites, higher stability and selectivity $[10,11]$. In this paper we have synthesized highly monodispersed PANI and hybrid Au@PANI nanocomposites by a facile in-situ polymerization method to study their gas sensor performance towards $\mathrm{NH}_{3}$ gas. Highly enhanced room temperature ammonia sensing properties of PANI and Au@ PANI nanospheres are reported for the detection of trace level (1-5 ppm) $\mathrm{NH}_{3}$ gas. The superior gas sensor performance of hybrid Au@ PANI nanospheres and gas/material interaction mechanism due to the presence of hybrid interfaces are reported.

\section{Materials and Methods}

Aniline $\left(\mathrm{C}_{6} \mathrm{H}_{5} \mathrm{NH}_{2}, 99.9 \%\right.$, Merck) was used as the monomer for synthesis of PANI which was distilled under reduced pressure before use. Tetrachloroauric acid trihydrate $\left(\mathrm{HAuCl}_{4} \cdot 3 \mathrm{H}_{2} \mathrm{O}, 99.999 \%\right.$ Sigma Aldrich) $\mathrm{HCl}$ (35.4\%, Merck) and $\mathrm{NaOH}$ (98\% Merck) were used for synthesis and used without any further purification. Ultrapure water ((Merck-Millipore, resistivity: $18.2 \mathrm{M} \Omega . \mathrm{cm})$ was used throughout the experiments.

\section{Synthesis of PANI and Au@PANI nanospheres}

For the synthesis [12] of uniform sized PANI nanospheres 1.0 mmol of aniline was dissolved in $100 \mathrm{~mL}$ of deionized water and then the acidity of the solution was adjusted to a certain $\mathrm{pH}$ (3) value as the initial $\mathrm{pH}$ value by the addition of trace amounts of $\mathrm{HCl}$ or $\mathrm{NaOH}$.

*Corresponding author: Pullithadathil B, Assistant Professor (Sr. Gr.) in Nanotechnology, Nanosensor Laboratory, PSG Institute of Advanced Studies Coimbatore - 641 004, India, Tel: +91 422 4344000, Extn (4193); E-mail: bijuja123@yahoo.co.in

Received May 01, 2015; Accepted May 18, 2015; Published June 01, 2015

Citation: Abdulla S, Dhakshanamoorthi J, Dinesh VP, Pullithadathil B(2015) Controlled Fabrication of Highly Monodispersed, Gold Nanoparticles Grafted Polyaniline (Au@PANI) Nanospheres and their Efficient Ammonia Gas Sensing Properties. J Biosens Bioelectron 6: 165. doi:10.4172/2155-6210.1000165

Copyright: ( 2015 Abdulla S, et al. This is an open-access article distributed under the terms of the Creative Commons Attribution License, which permits unrestricted use, distribution, and reproduction in any medium, provided the original author and source are credited. 
Citation: Abdulla S, Dhakshanamoorthi J, Dinesh VP, Pullithadathil B(2015) Controlled Fabrication of Highly Monodispersed, Gold Nanoparticles Grafted Polyaniline (Au@PANI) Nanospheres and their Efficient Ammonia Gas Sensing Properties. J Biosens Bioelectron 6: 165. doi:10.4172/2155-6210.1000165

Page 2 of 5

Similarly, $0.3 \mathrm{mmol}$ of $\mathrm{HAuCl}_{4}$ was dissolved in $100 \mathrm{~mL}$ of deionized water and the acidity of the solution was adjusted to the corresponding $\mathrm{pH}$ (3) value. Subsequently, these two solutions were mixed rapidly at room temperature and the reaction was allowed to proceed without any disturbance for $24 \mathrm{~h}$. The product was centrifuged at $2500 \mathrm{rpm}$ to remove the gold agglomerates formed as a by-product. The upper liquid phase was centrifuged at $7000 \mathrm{rpm}$ to obtain the PANI precipitate. Finally, the resulting PANI was washed with deionized water and ethanol several times to remove residual reactant and dried under vacuum at $50^{\circ} \mathrm{C}$ for $24 \mathrm{~h}$. For the synthesis of Au@PANI, the reaction mixture (aniline $+\mathrm{HAuCl}_{4}$ ) was continuously stirred for additional $24 \mathrm{~h}$ which led to the formation of Au nanoparticles adhered on the surface of PANI nanospheres due to the surface nucleation of Au under vigorous continuous stirring condition.

\section{Characterization techniques}

The purified PANI and Au@PANI nanospheres were characterized using various spectroscopic and microscopic techniques. UVVis absorption spectra were acquired using T90+ UV-Visible spectrophotometer (PG Instruments, UK). FT-IR spectra of the samples were recorded using Nicolet Impact 400 FT-IR spectrophotometer using $\mathrm{KBr}$. The structure and morphology of the nanocomposite were examined using High-Resolution Transmission Electron Microscopy (JEOL JEM-2010, HRTEM, Japan) in-built with energy-dispersive $\mathrm{X}$-ray spectrometer (Oxford INCA, Instruments, UK).

\section{Gas sensor fabrication and testing}

For testing and calibration of $\mathrm{NH}_{3}$ gas sensor, gold sputtered inter digitated array electrode (IDA) was used. The PANI and Au@PANI nanospheres were then dispersed in ethanol and sonicated for $15 \mathrm{~min}$ prior to coating on to the IDA electrode. The material was further spincoated (1500 rpm for $60 \mathrm{~s}$ ) onto the IDA electrodes and dried at $60^{\circ} \mathrm{C}$ for $30 \mathrm{~min}$. The gas sensing experiments of PANI and Au@PANI nanospheres were performed by monitoring the changes in resistance $(\Delta \mathrm{R})$ of the sensor upon exposure of $\mathrm{NH}_{3}$ gas. Nitrogen $\left(\mathrm{N}_{2}\right)$ was used as the carrier gas for all experiments. The schematic illustration of the experimental setup used is shown in Figure 1. The sensor was placed inside a double-walled stainless steel sensing chamber and the resistance change was monitored as a function of adsorbed gas at room temperature using an Agilent 34401A digital multimeter which is connected to a computer by USB interface for data acquisition.

\section{Results and Discussion}

For the enhancement in the gas sensing property analysis, the morphology of nanostructured PANI plays an active role compared to its bulk counterparts. In the present work, monodispersed nanospheres of PANI and Au@PANI were prepared in order to enhance the effective surface area of PANI. The PANI and Au@PANI nanospheres were synthesized using $\mathrm{HAuCl}_{4}$ as an oxidant for polymerization. Ammonium peroxydisulfate (APS) was used as an oxidant in the chemical synthesis of PANI. In contrast, when $\mathrm{HAuCl}_{4}$ was used as an oxidant the morphological evolution was observed in the PANI under various $\mathrm{pH}$ conditions. It is well known that the standard oxidation/ reduction potential of $\mathrm{HAuCl}_{4}(0.994 \mathrm{~V})$ is lower than that of APS $(2.0$ $\mathrm{V})$ which allows the controlled growth of PANI nanostructures [1316]. Hence, reaction time was kept for longer period in the presence of $\mathrm{HAuCl}_{4}$ which might be helpful in hindering the process of elongation of nanospheres into nanotubes or nanofibers and will only lead to the formation of monodispersed, uniform PANI nanospheres [13]. The formation of PANI and Au@PANI nanospheres could be explained

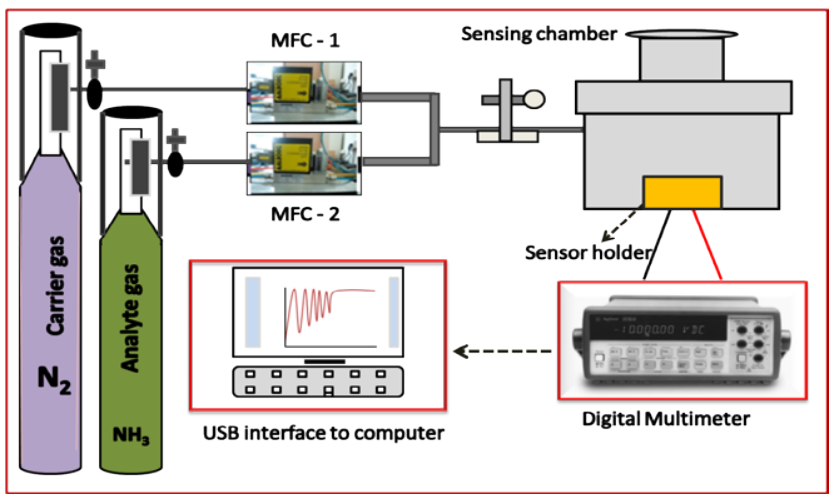

Figure 1: Schematic diagram of the experimental setup used for gas sensor performance analysis.

on the basis of nucleation theory reported by Jiahua Shi, Aniline is an organic base with $\mathrm{pKa}=4: 6$ at $25^{\circ} \mathrm{C}$. At different $\mathrm{pH}$-initial aqueous solutions, the anilinium ions and neutral aniline molecules coexist in different proportions, leading to different PANI nanostructures. In the present work initial $\mathrm{pH}$ value was set to 3 . At $\mathrm{pH}$ initial $=3$, anilinium ions and neutral aniline molecules coexist in different proportions. The anilinium ion shows good solubility, while neutral aniline molecules will form nanodroplets due to the limited miscibility in water and these aniline molecules are easier to oxidize since the electron pair on nitrogen is delocalized in neutral aniline molecules rather than localized in the anilinium ion $[10,13,17]$. When $\mathrm{HAuCl}_{4}$ is added to the aniline solution, the heterogeneous nucleation will first take place at the interface of water/aniline nanodroplets which would act as a template guiding the formation of PANI nanospheres and then anilinium ions in the bulk solution would subsequently participate in the PANI growth at the surface of these nanospheres to form solid PANI nanospheres [18]. In the case of Au@PANI, due to the vigorous stirring, nucleation of $\mathrm{Au}(0)$ happens on the surface of PANI nanospheres and gradually grow into nanoclusters constituting the Au@PANI nanospheres.

\section{UV-Vis spectroscopic analysis}

UV-Vis spectrum of PANI shows characteristics peaks at $329 \mathrm{~nm}$, $435 \mathrm{~nm}$ which are associated with $\pi-\pi^{*}$ and $\pi$-polaron transitions respectively $[11,12]$. Here, to the $\pi$-polaron transitions are disappeared and $\mathrm{n}-\pi^{*}$ absorbance was observed at $560 \mathrm{~nm}$ which is associated with a benzenoid to quinoid excitonic transitions [13] (Figure 2a). UV-Vis spectra of Au@PANI nanospheres shows characteristics peaks at $338 \mathrm{~nm}, 554 \mathrm{~nm}$ which is blue shifted in comparison with the PANI nanospheres (Figure $2 \mathrm{~b}$ ). The shift in the $\pi-\pi^{*}$ and $\pi$-polaron transitions clearly indicates the interaction between PANI and Au. The characteristic peak of Au NPs appears generally at $510 \mathrm{~nm}$ which is caused by the surface plasmon resonance. But the plasmon band of $\mathrm{Au}$ was not observed in the composite which might be due to the reduction of absorption intensity by the surrounding PANI. This result further suggests that, $\mathrm{Au}$ NPs has been adsorbed in to the PANI structure efficiently [4].

\section{FT-IR spectroscopic analysis}

The chemical structure of the as prepared PANI and Au@PANI nanospheres were identified using FT-IR analysis. Figure 3 depicts the FT-IR spectra of PANI and Au@PANI nanospheres synthesized at initial $\mathrm{pH}$ value of 3. In the spectra of PANI nanospheres, peaks at 1592 and $1495 \mathrm{~cm}^{-1}$ were identified which are the characteristics peaks of 
Citation: Abdulla S, Dhakshanamoorthi J, Dinesh VP, Pullithadathil B(2015) Controlled Fabrication of Highly Monodispersed, Gold Nanoparticles Grafted Polyaniline (Au@PANI) Nanospheres and their Efficient Ammonia Gas Sensing Properties. J Biosens Bioelectron 6: 165. doi:10.4172/2155-6210.1000165

Page 3 of 5

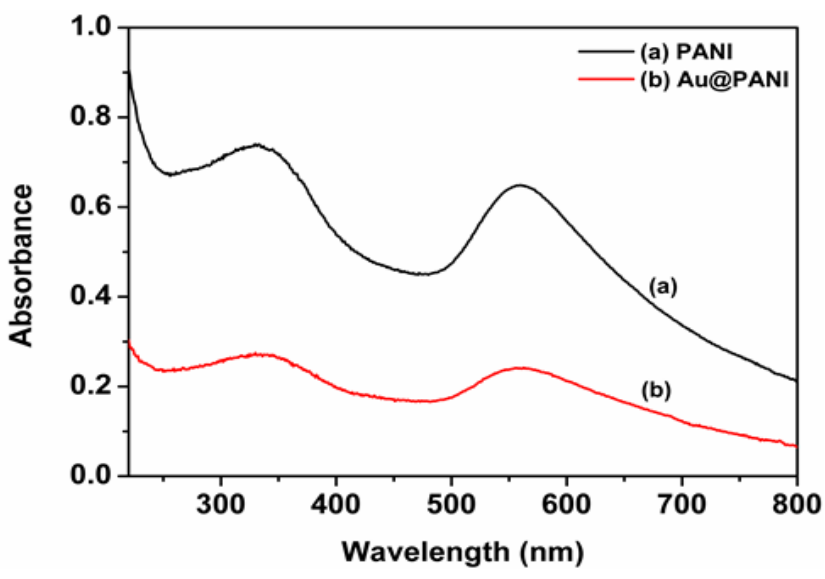

Figure 2: UV-Vis spectrum of (a) PANI nanospheres (b) Au@PANI nanospheres synthesized at initial $\mathrm{pH}$ value of 3 .

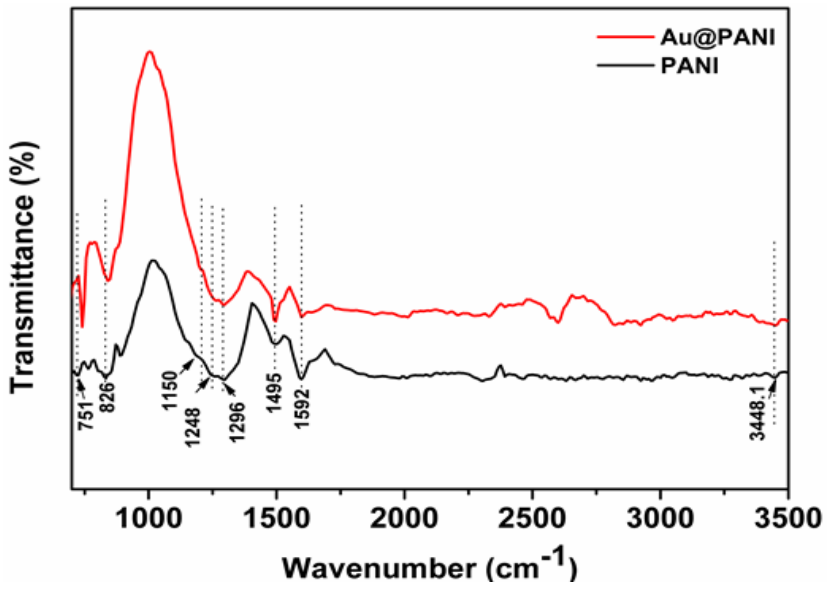

Figure 3: FT-IR spectra of (a) PANI nanospheres (b) Au@PANI nanospheres at initial $\mathrm{pH}=3$.

PANI corresponding to $t$ the $\mathrm{C}=\mathrm{C}$ stretching of quinoid and benzenoid rings respectively. The peaks at 1296 and $1248 \mathrm{~cm}^{-1}$ are attributed to the $\mathrm{C}-\mathrm{N}$ (of the secondary aromatic amines) and $\mathrm{C}=\mathrm{N}$ stretching modes. The peak appeared at $1150 \mathrm{~cm}^{-1}$ is assigned to the in-plane bending of aromatic $\mathrm{C}-\mathrm{H}$ which is also a considered as a measure of the degree of delocalization of electrons in PANI and it is directly related to the degree of doping and electrical conductivity of PANI [11,12-15].

The characteristic N-H stretching mode was found to be present at $3448,3317 \mathrm{~cm}^{-1}$. The peaks observed at $826 \mathrm{~cm}^{-1}, 751 \mathrm{~cm}^{-1}$ was attributed to the out-of-plane bending of $\mathrm{C}-\mathrm{H}$ in the 1,4-disubstituted benzene ring) and vibrations of the monosubstituted benzene ring respectively [13]. The peaks observed for PANI nanospheres are in good agreement with the reported value for PANI. In the FT-IR spectra of Au@PANI nanospheres the similar peaks were observed confirming the presence of PANI in the composites.

\section{Transmission electron microscopic analysis}

The morphological studies of PANI and Au@PANI nanospheres were carried out using High resolution Transmission Electron Microscopy (HRTEM). Figure 4 depicted the TEM analysis of the PANI and Au@PANI nanospheres. The formation of nanospheres of
PANI is well evident from the Figure $4 \mathrm{a}$ and $4 \mathrm{~b}$. The PANI nanospheres were observed to be highly uniform and monodispersed. The diameter of the spheres was found to be $\sim 63-64 \mathrm{~nm}$.

The EDS spectrum of PANI nanospheres is depicted in Figure 4c. The spectrum strongly revealed the presence of pure PANI containing C, $\mathrm{N}$ and traces of $\mathrm{Au}$ signatures. The formation of Au@PANI composites were well cleared from the TEM analysis. Figure $4 \mathrm{~d}$ and e shows the TEM images of Au@PANI nanospheres. The average diameter of the $\mathrm{Au}$ nanoparticles was found to be $\sim 25 \mathrm{~nm}$. EDS spectrum in Figure $4 \mathrm{f}$ shows strong signatures of Au compared to that of PANI indicating the nanocomposite formation. It is observed that, during the growth of the PANI spheres, Au nanoparticles are well dispersed in the PANI. In the initial stage of the synthesis, the seed-mediated growth of $\mathrm{Au}$ was attained followed by the reduction to the Au nanoparticles. Hence, simultaneous growth of $\mathrm{Au}$ and PANI spheres were observed in the composites and each PANI spheres were found to have bond with $\mathrm{Au}$ nanoparticles suggesting the fact that, partial oxidation and reduction was happened during the composite formation. .

\section{Gas sensing performance analysis}

The gas sensing performance of the PANI and Au@PANI nanospheres towards trace-level $\mathrm{NH}_{3}$ gas under ambient conditions have been studied. Initially, PANI and Au@PANI nanospheres were spin coated on sputtered gold IDA electrodes and the electrical resistance of both the sensors were measured initially at room temperature under atmospheric pressure conditions. The enhancement in the electrical properties of PANI and Au@PANI nanospheres was studied by performing the current-voltage $(I-V)$ characteristics using two-probe technique. Figure 5 represents $I-V$ curves of the PANI and $\mathrm{Au} @ \mathrm{PANI}$ nanospheres recorded at room temperature. Both the PANI and Au@PANI based sensor exhibited ohmic behaviour within the range of -1 to $+1 \mathrm{~V}$.

In chemiresistive based sensors, the response and recovery characteristics depend on the rate of diffusion of the analyte gas molecules and the subsequent reaction between the sensing materials. The gas sensing response of the PANI and Au@PANI nanospheres based sensors was analyzed for various concentrations analyte molecules. The change in the resistance was measured upon exposure to $\mathrm{NH}_{3}$ gas molecules. The dynamic responses of the sensor materials, their reversibility, and detection limits towards $\mathrm{NH}_{3}(3 \mathrm{ppm})$ were studied at room temperature. Figure $6 \mathrm{a}$ and $\mathrm{b}$ ) shows the response and

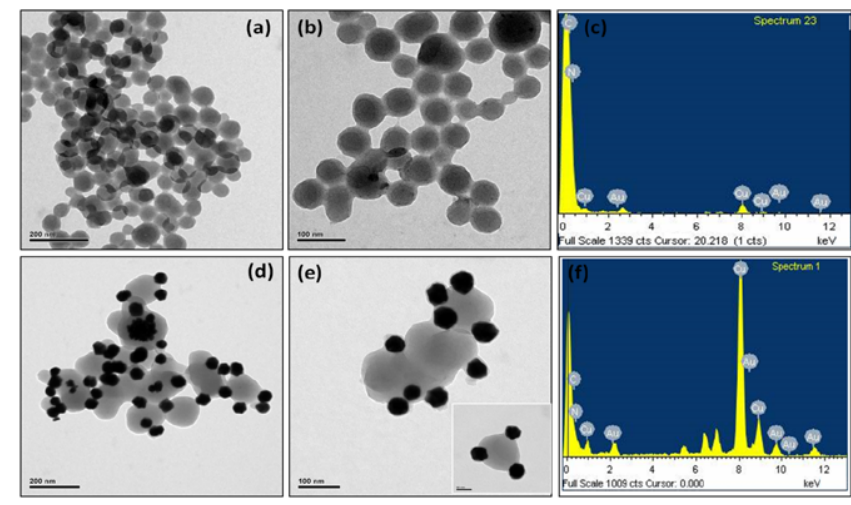

Figure 4: HRTEM images of (a-b) PANI nanospheres and (d-e) Au@PANI nanospheres, $(c, f)$ corresponding EDS spectra. 
Citation: Abdulla S, Dhakshanamoorthi J, Dinesh VP, Pullithadathil B(2015) Controlled Fabrication of Highly Monodispersed, Gold Nanoparticles Grafted Polyaniline (Au@PANI) Nanospheres and their Efficient Ammonia Gas Sensing Properties. J Biosens Bioelectron 6: 165. doi:10.4172/2155-6210.1000165

Page 4 of 5

recovery characteristics of the PANI and Au@PANI nanospheres based sensor towards the exposure of $3 \mathrm{ppm}$ of $\mathrm{NH}_{3}$.

As presented in Figure 6 the response time $\left(\mathrm{T}_{90}\right)$ of the PANI nanospheres sensor was calculated to be $36 \mathrm{~s}$ whereas, for Ag@PANI nanospheres based sensor it was found to be $61 \mathrm{~s}$ for $3 \mathrm{ppm}$ of $\mathrm{NH}_{3}$. The sensitivity of the Ag@PANI nanospheres based sensor were found to be enhanced compared to PANI nanospheres (Scheme 1).

Figure $7 \mathrm{a}$ and $\mathrm{b}$ represents the dynamic sensor response graph of PANI and Au@PANI nanospheres towards the trace-level concentrations of ammonia gas $(2-10 \mathrm{ppm})$ under ambient conditions. From the results, it is evident that, the sensor resistance of both the sensors increased immediately upon exposure to target gas. The sensor was found to be completely recoverable at room temperature suggesting the physisorption of gas molecules with the sensing materials. Significant improvement in the sensitivity of the Au@PANI nanospheres was observed upon exposure to different concentrations of $\mathrm{NH}_{3}$ gas compared to PANI nanospheres. For example, the sensitivity of the PANI nanospheres was found to be $20.2 \%$. Whereas, Au@PANI nanospheres based sensor exhibited higher sensitivity of 50.2\% (Figure $7 \mathrm{c})$. The sensor was tested under various oxidizing and reducing gases such as $\mathrm{NO}_{2}, \mathrm{H}_{2} \mathrm{~S}, \mathrm{C}_{2} \mathrm{H}_{5} \mathrm{OH}, \mathrm{C}_{5} \mathrm{H}_{8}$. Figure $7 \mathrm{~d}$ depicts the selectivity result of the Au@PANI nanospheres at concentration of 2 ppm of each analyte gas. It was observed that, Au@PANI nanospheres based sensor was highly selective towards ammonia (62.3\%) compared to the other interfering gases. The observed enhancement in the gas sensing performance of the Au@PANI nanospheres could be attributed to the

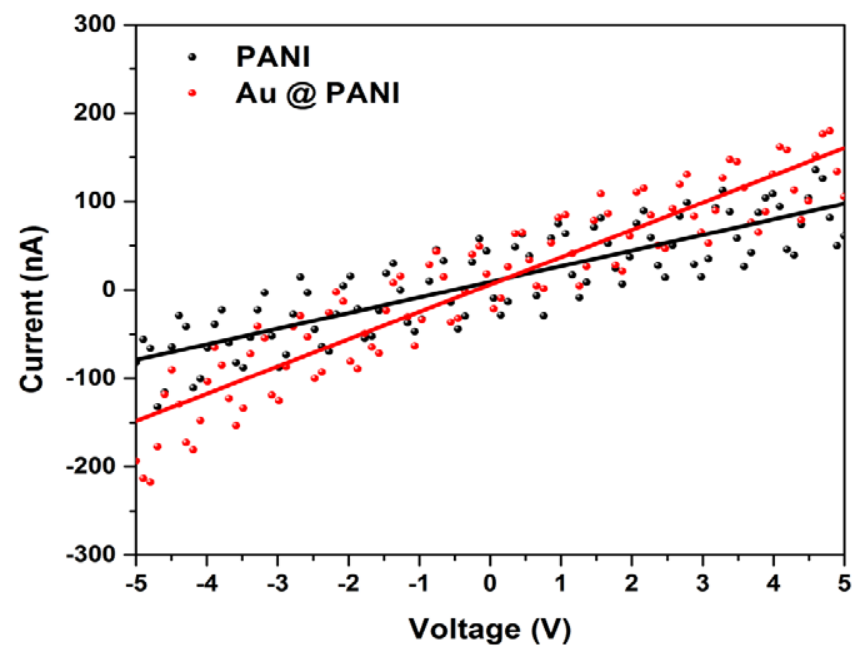

Figure 5: I-V characteristics of PANI and Au@PANI nanospheres.
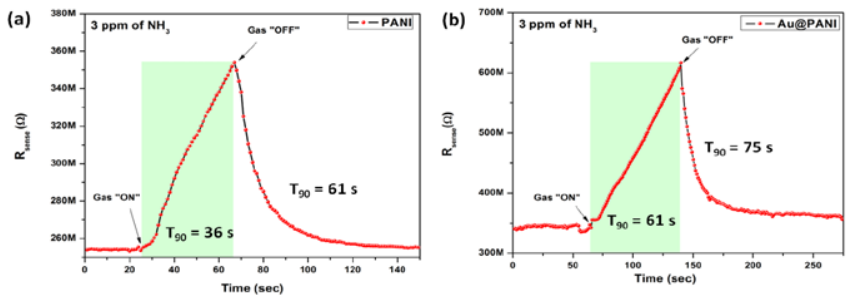

Figure 6: (a) Response (b) recovery time of the PANI and Au@PANI nanospheres towards exposure towards $3 \mathrm{ppm}$ of $\mathrm{NH} 3$.

\begin{tabular}{|c|c|c|c|c|c|c|}
\hline $\begin{array}{c}\text { Concentration } \\
\text { (ppm) }\end{array}$ & \multicolumn{2}{|c|}{$\begin{array}{c}\text { Sensitivity } \\
\text { ( } \Delta \text { R/RA \%) }\end{array}$} & \multicolumn{2}{|c|}{ Response Time (s) } & \multicolumn{2}{|c|}{$\begin{array}{c}\text { Recovery Time } \\
\text { (s) }\end{array}$} \\
\hline & PANI & Au@PANI & PANI & Au@PANI & PANI & Au@PANI \\
\hline $\mathbf{1}$ & 20 & 50.2 & 21 & 48 & 46 & 58 \\
\hline $\mathbf{2}$ & 30.6 & 62.3 & 26 & 55 & 55 & 71 \\
\hline $\mathbf{3}$ & 39.2 & 79.4 & 36 & 61 & 61 & 75 \\
\hline $\mathbf{4}$ & 56.9 & 98.5 & 50 & 75 & 66 & 90 \\
\hline $\mathbf{5}$ & 76.1 & 108.7 & 66 & 83 & 101 & 114 \\
\hline
\end{tabular}

Table 1: Comparison of sensitivity, response time, recovery time of PANI and Au@PANI nanospheres.
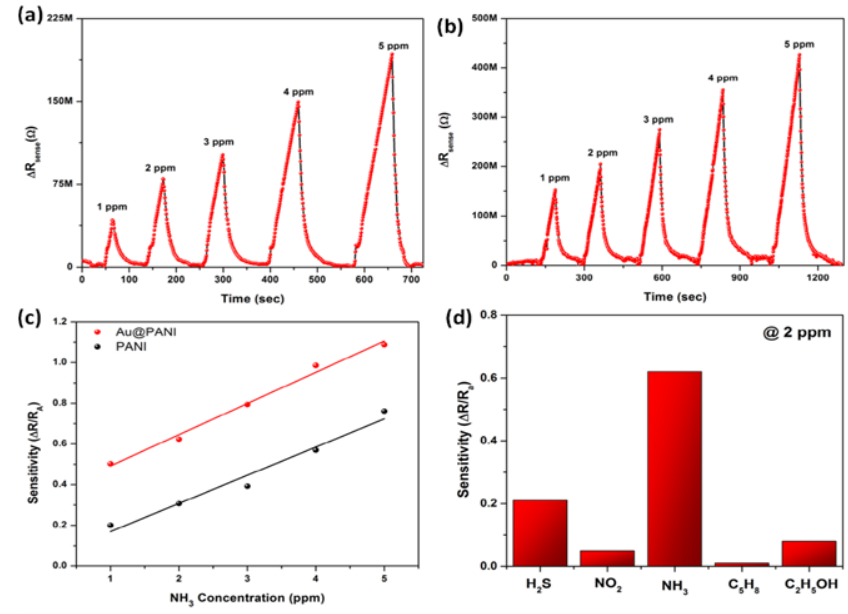

Figure 7: Dynamic response of (a) PANI nanospheres (b) Au@PANI nanospheres under various concentrations of ammonia (1-5 ppm) (c) sensitivity of the PANI and Au@PANI nanospheres and (d) selectivity result of the Au@ PANI nanospheres.

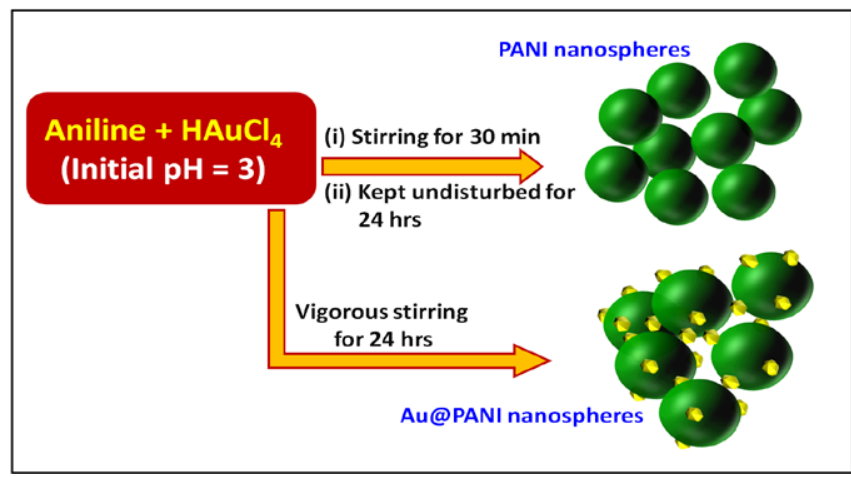

Scheme 1: Synthesis of PANI and Au@PANI nanospheres at initial pH value of 3.

following reason. (i) increased surface area of the nanospheres, which provided large number of active sites for ammonia adsorption. (ii) controlled doping of Au nanoparticles in the PANI nanospheres which created an easy pathway for charge transfer. The sensing mechanism of PANI with $\mathrm{NH}_{3}$ is well understood [19]. It is mainly governed by the combination of protonation/deprotonation reaction in PANI. PANI is a $p$-type semiconductor. On exposure to the electron donating ammonia gas molecules, the proton on the $\mathrm{N}^{+}-\mathrm{H}$ groups of PANI and forms $\mathrm{NH}_{4}^{+}$ions and lead to the localization of polarons of conducting PANI as represented in equation (1). This causes protonation in PANI 
Citation: Abdulla S, Dhakshanamoorthi J, Dinesh VP, Pullithadathil B(2015) Controlled Fabrication of Highly Monodispersed, Gold Nanoparticles Grafted Polyaniline (Au@PANI) Nanospheres and their Efficient Ammonia Gas Sensing Properties. J Biosens Bioelectron 6: 165. doi:10.4172/2155-6210.1000165

and increases its electrical resistance. When the sensor is exposed to the fresh air again, the $\mathrm{NH}_{4}^{+}$ions decompose into $\mathrm{NH}_{3}$ molecules and protons. This reversible transformation results in restoring the initial degree of doping and the resistance of the sensor material [19]. In the presence of Au nanoparticles, on the surface of the PANI, due to surface doping $\mathrm{Au}$ could interact with $\mathrm{NH}_{3}$ and transfer electrons to the PANI. The Au doping has also could increase the surface area of the polymer and will provide large number of active sites for ammonia adsorption. It is plausible to conclude that, the synergetic effect between the metal nanoparticles and conducting polymer had resulted in better performance compared to its individual component. The sensors were observed to be completely recoverable after each exposure towards ammonia at room temperature. Consolidated results of the response and recovery time of both sensors are summarized in Table 1.

\section{Conclusion}

A novel facile template free (in-situ polymerization of aniline) chemical approach method was adopted for the synthesis of PANI and hybrid Au@PANI nanospheres. Monodispersed polyaniline with an average diameter of $64 \pm 0.5 \mathrm{~nm}$ was observed from TEM studies, and its characteristic $\pi$-polaron transition in UV-visible spectrum suggesting the as-synthesised polyaniline exist in emeraldine base form. FT-IR studies of polyaniline supports it strongly for the existence of emeraldine form with its characteristic $\mathrm{C}=\mathrm{C}, \mathrm{C}-\mathrm{N}$ and $\mathrm{C}=\mathrm{N}$ stretchings. The peak appearing at $1150 \mathrm{~cm}^{-1}$ assigned to the inplane bending of aromatic $\mathrm{C}-\mathrm{H}$ considered as a measure of the degree of delocalization of electrons in PANI and was directly related to the degree of doping and electrical conductivity of PANI. Similarly Hybrid Au@PANI nanospheres prepared by in-situ polymerization shows the characteristic $\pi$-polaron in UV-visible studies along with the surface plasmon peak of $\mathrm{Au}$ nanospheres thus proving the formation of hybrid Au@PANI nanospheres with electron transfer process. TEM observation clearly suggests the decoration of $\mathrm{Au}$ nanospheres $(25$ $\pm .5 \mathrm{~nm}$ ) over the PANI spheres thereby increasing the conjugation and formation of interfaces between $\mathrm{Au}$ and PANI nanospheres. Comparative $\mathrm{NH}_{3}$ gas sensing evaluation of PANI and Au@PANI nanospheres exhibit an enhanced sensing for hybrid Au@PANI nanospheres for trace level analyte molecules in the range of 1-5 ppm under ambient conditions. The superior sensing behaviour of Au@ PANI nanospheres with high sensitivity (58.2\%), rapid response (48 s) and recovery ( $58 \mathrm{~s}$ ) characteristics are due to the synergetic effect of $\mathrm{Au}$ and PANI nanospheres at the interface, thus proves to a potential $\mathrm{NH}_{3}$ sensor device fabrications in near future.

\section{Acknowledgements}

The authors wish to acknowledge the facilities and support provided by the management, PSG Sons and Charities, Coimbatore. Author, AS acknowledge UGC-Maulana Azad National Fellowship (MANF), New Delhi, INDIA for fellowship. The author DJ acknowledges CSIR, New Delhi, INDIA for fellowship.

\section{References}

1. David WH, Mira J (2008) Composites of intrinsically conducting polymers as sensing nanomaterials. Chem Rev 108: 746-769.

2. Junsheng W, Jixiao W, Zhi W, Fengbao Z (2009) A Template-Free Method toward Urchin-Like Polyaniline Microspheres. Macromol Rapid Commun 30: 604-608.
3. Wenbin Z, Yongxin W, Yan Y, Yufeng S, Jianping D, et al. (2007) Fabrication of Shape-Controllable Polyaniline Micro/Nanostructures on Organic Polymer Surfaces: Obtaining Spherical Particles, Wires, and Ribbons. J Phys Chem B 111: 3918-3926.

4. Xiaomiao F, Changjie M, Gang Y, Wenhua H, Jun-Jie Z (2006) Polyaniline/Au Composite Hollow Spheres: Synthesis, Characterization, and Application to the Detection of Dopamine. Langmuir 22: 4384-4389.

5. Kailash RN, Sandeep A. Waghuley (2013) Gas Sensing Mechanism of Metal Oxide Doped PANi Composites. J Mater Sci Eng B 3: 310-313.

6. Guerra-Balcázar M, Ortega R, Castaneda F, Arriaga L.G, LedesmaGarcia J (2011) Synthesis of Au Nanoparticles/Polyaniline Composites by Electroreduction for Glucose Oxidation. Int J Electrochem Sci 6: 4667-4675.

7. Lifang H, Yong J, Fanli M, Minqiang L, Jinhuai L (2009) Gas sensors for ammonia detection based on polyaniline-coated multi-wall carbon nanotubes. J Mater Sci Eng B 163: 76-81.

8. Debarnot DN, Poncin-Epaillard F (2003) Polyaniline as a new sensitive layer for gas sensor. Analytica Chimica Acta 475: 1-15.

9. Kaushik M, Mike JW, Andy D, Mike SS (2005) Polymerization of Aniline by Auric Acid: Formation of Gold Decorated Polyaniline Nanoballs. Macromol Rapid Commun 26: 232-235.

10. Stejskal J, Sapurina I, Trchova M, Konyushenko EN (2008) Oxidation of aniline: polyaniline granules, nanotubes, and oligoaniline microspheres. Macromolecules 41: 3530-3536.

11. Iole V, llaria F, Maria Vittoria R, Andrea B (2013) A nanostructured composite based on polyaniline and gold nanoparticles: synthesis and gas sensing properties. Nanotechnology 24: 155503.

12. Neoh KG, Kang ET, Tan KL (1993) Evolution of polyaniline structure during synthesis. Polymer 34: 3921-3928.

13. Jiahua S, QiangW, Runming L, Yinxu Z, Yujun Q, et al. (2013) The pH controlled morphology transition of polyaniline from nanofibers to nanospheres. Nanotechnology 24: 175602

14. Wang Y, Liu Z, Han B, Sun Z, Huang Y, et al. (2005) Facile synthesis of polyaniline nanofibers using chloroaurate acid as the oxidant. Langmuir 21 833-836.

15. Zhang L, Peng H, Kilmartin PA, Soeller C, Tilley R, et al. (2008) Self-assembled hollow polyaniline/Au nanospheres obtained by a one-Step synthesis. Macromol Rapid Commun 29: 598-603.

16. Weast RC, Astle MJ (1981) CRC Handbook of Chemistry and Physics (61st edn) Boca Raton, CRC Press, Florida, USA

17. Fu Y, Elsenbaumer RL (1994) Thermochemistry and kinetics of chemical polymerization of aniline determined by solution calorimetry. Chem Mater 6 : 671-677.

18. Ding H, Shen J, Wan M, Chen Z (2008) Formation Mechanism of Polyaniline Nanotubes by a Simplified Template-Free Method. Macromol Chem Phys 209 : 864-871.

19. Wojkiewicza JL, Bliznyukc VN, Carquignya S, Elkamchia N, Redona N, et al. (2011) Nanostructured polyaniline-based composites for ppb range ammonia sensing. Sens Actuators B 160: 1394-1403. 